\title{
Stigma a major barrier to treatment for pregnant women with addictions
}

$\mathrm{D}$ octors and other health care providers need to check their moral outrage at the door and treat pregnant women addicted to drugs and alcohol with compassion and supportive, team-based care, urges the Canadian Centre on Substance Abuse.

Stigma is one of the biggest barriers to effective treatment for pregnant women who use legal or illegal substances, according to the centre's new report, Licit and Illicit Drug Use During Pregnancy: Maternal, Neonatal and Early Childhood Consequences.

Pregnant women's use of tobacco, alcohol and opiods is "a burgeoning epidemic throughout the world," says Dr. Loretta Finnegan, the report's author. Finnegan is the former medical adviser to the director of the Office of Research on Women's Health at the US National Institutes of Health.

"Rather than discouraging discussions of drug use during pregnancy, we should be looking upon this as an opportunity to bring about positive, long-lasting change in the life of the mother and child, through effective treatment and support services," Finnegan told a Nov. 4 news conference in Ottawa, Ontario.

Instead of getting supportive care, many pregnant women who turn up at

clinics or emergency departments for help with their addictions feel unwelcome and judged, said Finnegan, who also founded a clinic for women with drug addictions in Philadelphia, Pennsylvania.

It is difficult to obtain accurate national figures on the number of women who use tobacco, alcohol or drugs during pregnancy because of underreporting. But the 2008 Canadian Perinatal Health Report, based on 2005 data, indicates that in the month prior to being surveyed, $13 \%$ of pregnant Canadian women reported smoking tobacco and $11 \%$ reported drinking alcohol. Another $5 \%$ of pregnant women also reported using illicit drugs during pregnancy, according to the Licit and Illicit Drug Use during Pregnancy report.

In the US, there are about 4.1 million babies born every year, and about $16 \%$ of pregnant women report past-month use of drugs or alcohol, Finnegan says.

Babies born to mothers dependent on drugs or alcohol may experience withdrawal symptoms (neonatal abstinence syndrome) once the umbilical cord is cut - even if their mothers take prescription drugs such as methadone or buprenorphine to help with withdrawal.

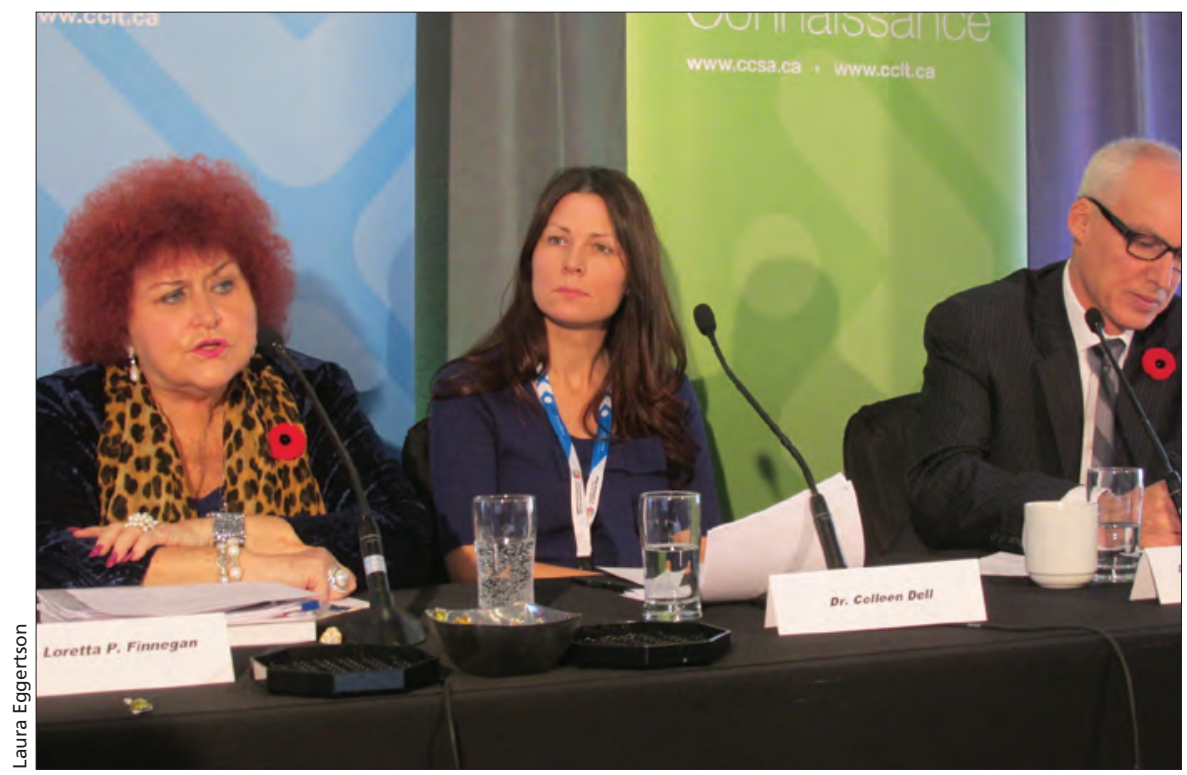

Dr. Loretta Finnegan, Colleen Dell and Franco Vaccarino introduced the Canadian Centre on Substance Abuse's new report on treating pregnant women with addictions.
Across Canada, there were 1057 cases reported of neonatal abstinence syndrome in 2009-2010, according to the Canadian Centre on Substance Abuse report First Do No Harm: Responding to Canada's Prescription Drug Crisis.

In Ontario, the number of babies born with the syndrome has been increasing, says Rita Notarandrea, the deputy CEO of the centre. In 2009-2010, there were 564 reported cases, up from 171 cases in 2003, according to First Do No Harm. That increase is driving concern for pregnant women to get treatment for their addictions, Notarandrea said.

Before women seek treatment, however, they need to be assured of informed, nonjudgmental care that will support their needs long after the babies are delivered, the report urges. Doctors need to be better informed about the unique treatment needs of women, who progress more quickly than men into alcohol and opioid dependence, as well as into related circulatory disorders, cirrhosis of the liver and hypertension. Women are also at greater risk than men of severe cocaine-related health problems.

"Pregnant women who smoke, who drink, who use prescription or illegal drugs are as deserving of compassion, comprehensive support, interventions and treatment - and perhaps even more so - as anyone else presenting with an addiction issue," Notarandrea told the news conference.

That's because two-thirds of women with substance abuse problems also have concurrent mental health problems, such as depression, anxiety and post-traumatic stress disorder, and a large proportion have been sexually and/or physically abused in childhood or as adults, says Colleen Dell, the research chair in substance abuse at the University of Saskatchewan in Saskatoon.

"So it's harmful for us to look at pregnant women with addiction issues and assume it's as simple as saying 'For the sake of the baby, stop using'," Dell said. — Laura Eggertson, CMAJ

CMAJ 2013. DOI:10.1503/cmaj.109-4653 